\title{
Rasgos de la excelencia en la investigación: proactividad, pasión por el conocimiento y resiliencia
}

\section{Proactivity, passion for knowledge, and resiliency, qualities to develop excellence in research}

\section{KARIN YOVANA QUIJADA LOVATÓN*}

El artículo analiza los rasgos personales que caracterizan a los profesores de la Universidad de Colima y la Universidad Autónoma de Sinaloa que han alcanzado una carrera exitosa en la investigación. En la literatura cualidades y experiencias académicas que tienen un gran impacto en el buen desempeño de la profesión, que se agrupan en expectativas de éxito, motivaciones y habilidades metacognitivas. En cada institución se seleccionaron, a través de un muestreo intencional, diez investigadores con trayectorias significativas, a quienes se les aplicó una entrevista semiestructurada. En el análisis de la información se empleó un enfoque comparativo cualitativo, que coadyuvó a organizar los resultados en tres conceptos clave: conducta proactiva, pasión por el conocimiento y resiliencia. Los principales hallazgos demuestran que estos rasgos influyen de manera positiva en el trabajo intelectual, pues fortalecen la autoestima y la creatividad para adaptarse a los cambios y asumir las exigencias del ámbito científico.

The article analyses the personal qualities that characterize the professors of the Universidad de Colima and the Universidad Autónoma de Sinaloa to achieved a successful career in research. The literature on the subject explains that there are some academic qualities and experiences which affecting their professional attainment positively; they could be grouped in three: success expectancies, motivation and metacognitive abilities. In each University was selected a group of ten experienced professors by an intentional qualitative sample. After they accepted voluntarily the interview, every professor answered a semi-structured questionnaire. Later, the interview's analysis was based on a comparative and qualitative approach which was useful for organizing the interview's information in three fundamental concepts: proactivity, passion for knowledge, and resiliency. The results show that personal and professional profiles affect their intellectual job positively because these reinforce their self-confidence and creativity; both qualities are indispensable for adapting and confronting the standard requirements of the scientific environment.

\author{
Palabras clave: \\ rasgos personales, \\ excelencia, \\ investigadores y \\ universidad
}

Recibido: 25 de abril de 2018. Aceptado para su publicación: 18 de junio de 2018. Recuperado de: https://sinectica.iteso.mx/index.php/SINECTICA/article/view/877 DOI: 10.31391/S2007-7033(2018)0051-011

*Doctora en Pedagogía por la Universidad Nacional Autónoma de México. Miembro de la Red Nacional de Investigadores en Educación y Valores y del proyecto Estudio de la Excelencia del Profesorado del Posgrado de la UNAM. Actualmente, en una estancia de investigación en El Colegio de México. Sus líneas de investigación son la educación y los valores. Correo electrónico: karinyo85@hotmail.com 


\section{INTRODUCCIÓN}

1 ste artículo se desprende de un proyecto de investigación que inició en E 2013 con el objetivo de conocer los factores personales, profesionales e institucionales que intervienen en la formación de la identidad profesional de académicos con una trayectoria reconocida en la investigación y que trabajan en instituciones estatales de México, como es el caso de la Universidad de Colima (UdeC) y la Universidad Autónoma de Sinaloa (UAS) (Quijada, 2017). En sus aulas se han formado algunos personajes ilustres de la ciencia, el arte y la cultura, que han servido de inspiración a las generaciones siguientes, como el científico Gerardo Chowell-Puente, egresado de la Facultad de Telemática de la UdeC, que obtuvo el Premio Nacional de la Juventud 2002 por su investigación sobre la epidemia SARS (Universidad de Colima, 2003), o el doctor Ambrocio Mojardín Heráldez, egresado de la Facultad de Psicología de la UAS, que cuenta con la distinción de Científico Mexicano de Impacto Mundial 2012 por la Agencia Internacional de Información Thomson Reuters y el Consorcio de Universidades Mexicanas (Universidad Autónoma de Sinaloa, 2012).

Ambos investigadores, con trayectorias académicas distintas, fueron estudiantes que hicieron frente a los problemas económicos y sociales que afectan a Colima y Sinaloa. No obstante, en sus biografías este escenario suele pasar inadvertido frente a sus ambiciones personales y su predilección por la vida intelectual. Al igual que buena parte de los profesores que buscan posicionarse en el ámbito científico, han encontrado la fórmula del éxito profesional en una formación rigurosa, con publicaciones en revistas indizadas y el acompañamiento de líderes o mentores eficaces, así como la obtención de una plaza de tiempo completo en una universidad reconocida y la participación en diferentes grupos y redes de investigación.

García-Romero (2012) y Góngora (2012) coinciden con algunos de estos criterios e insisten en que el éxito académico y el acceso a un buen empleo y remuneraciones económicas dependen de la calidad de las instituciones educativas y las experiencias de aprendizaje que los sujetos adquieran a lo largo de su formación. En México, por ejemplo, desde finales de la década de los ochenta se comenzaron a impulsar una serie de reformas encaminadas a elevar la calidad de la enseñanza en las universidades públicas. Una de las medidas más relevantes fue la creación del Consejo Nacional de Ciencia y Tecnología (Conacyt), el Sistema Nacional de Investigadores (SNI) y el Programa para Desarrollo Profesional Docente (Prodep). Estos tenían la finalidad de promover el desarrollo científico y tecnológico en el país a través de la difusión de becas y subsidios para la investigación, la contratación de profesores de tiempo completo con doctorado y la reactivación del trabajo colegiado mediante la conformación de cuerpos académicos (Galaz et al., 2012).

Este proceso no fue ajeno a la UdeC y la UAS, que implementaron estas políticas de manera paulatina con el objetivo de mejorar su infraestructura, la calidad de sus programas educativos y formar profesionales competitivos. El profesorado, a su vez, se vio obligado a contar con un perfil en investigación acorde con las exigencias del nuevo milenio, así como evaluar su productividad periódicamente. La modernización en las dos instituciones no se hizo esperar, pues, de acuerdo con el informe anual de la Asociación Nacional de Universidades e Instituciones de Educación Superior (ANUIES), en 2016 se posicionaron en el segundo lugar con el 
mayor número de profesores de tiempo completo y miembros del SNI de la región centro occidente y noroeste de México (ANUIES, 2017).

A pesar de estos avances, estas instituciones aún presentan rezagos en el área científica. En la UdeC, por ejemplo, de los 34 programas de posgrado que se han creado en los últimos diez años, solo $44.1 \%$ se encuentran adscritos al Programa Nacional de Posgrados de Calidad y, de ellos, ocho son de doctorado (Universidad de Colima, 2018). Algo similar sucede en la UAS, pues de los 77 programas de posgrado, solo $24 \%$ son de doctorado (Universidad Autónoma de Sinaloa, 2017).

Los testimonios de un grupo significativo de investigadores de la UdeC y la UAS que participan en este estudio describen con mayor detalle estas necesidades y dificultades. Sin embargo, lejos de representar un obstáculo para alcanzar sus metas, para muchos de ellos constituye el principal aliciente que los ha "impulsado a buscar becas", "realizar estudios en el extranjero" o "gestionar la creación de institutos y redes de investigación". Si bien en sus relatos se advierten disimiles estrategias -personales y grupales- que les han permitido alcanzar resultados sobresalientes en su profesión, también se identifican cualidades e inquietudes comunes que los motivan a dedicar gran parte de su tiempo y esfuerzo a la construcción de nuevos conocimientos.

Al respecto, Bain (2012) señala que las buenas calificaciones, las clases magistrales y un sistema universitario óptimo no son suficientes, en ocasiones, para lograr la excelencia, pues se requiere talento, sistematicidad y pensamiento reflexivo para afrontar los desafíos del campo laboral. La realización de este artículo se inserta en esa línea; al margen de conocer los puntajes que algunos profesores obtienen en sus evaluaciones y las distinciones que han acumulado a nivel profesional, buscamos conocer qué rasgos de su personalidad han influido en su inclinación por la investigación y les han permitido hacer frente a las carencias y problemas económicos que aquejan a sus regiones.

Los estudios sobre el tema en Estados Unidos, España, Francia y México enumeran una serie de atributos cognitivos, emocionales y psicológicos que coadyuvan a que buena parte de los investigadores busquen mejores opciones educativas y potencien su desempeño académico (Hirsch, 2008; Visser-Wijnveen, Stes \& Petegem, 2014; Day y Gu, 2012; Moè, 2016). Los conceptos más empleados en este campo hacen referencia a siete elementos clave: autoeficacia, autoestima, motivación, interés, pasión, esfuerzo y competencia que, desde diferentes enfoques y corrientes epistémicas, promueven el bienestar personal y la satisfacción laboral en el profesorado (Skaalvik \& Skaalvik, 2009; Galaz, 2002; Gu \& Day, 2007).

A pesar de que estos rasgos juegan un papel central en su desarrollo profesional, no los exentan de la frustración y el desánimo; todo lo contrario, los alientan a mejorar día con día. Asimismo, no son cualidades exclusivas de los sujetos de estudio, sino que todos las poseemos, aunque no en el mismo nivel de condiciones, ya que algunos presentan mayor predisposición para ciertas actividades intelectuales que otros. Sin embargo, la diferencia principal reside en el modo en que se emplean y el grado de confianza que han alcanzado de sus capacidades, pues, al tener una imagen positiva de sus logros y fortalezas, presentan un mejor manejo de sus emociones (Eccles \& Wigfield, 2002; Vallerand et al., 2003). 


\section{MARCO TEÓRICO}

En los últimos veinte años, las investigaciones que abordan el tema de los académicos y su formación se han interesado en identificar y analizar los rasgos y cualidades de la personalidad que influyen en el buen ejercicio de la docencia y la investigación (Bain, 2004; Day, 2006, Martínez-Navarro, 2011). Al respecto, los trabajos de Beijaard, Verloop y Vermunt $(2000,2004)$, Day, Kington, Stobart y Sammons (2006) y Canrinus Helms-Lorenza, Beijaard, Buitinka y Hofmana (2011) revelan experiencias y atributos personales que coadyuvan a explicar cómo el pasado educativo y las actividades fuera de las universidades impactan en el modo en que perciben su trabajo y el compromiso que manifiestan hacia su desarrollo profesional. Ambos autores coinciden en que, en este ámbito, los sujetos comparten ciertas motivaciones, intereses y metas que guían, de múltiples maneras, su vocación por la ciencia.

En esa misma perspectiva, Badia, Meneses y Monereo (2014) hacen un recuento de las principales teorías en torno a los sentimientos y emociones que experimentan los profesores en el plano laboral, así como el valor que le otorgan a la enseñanza en la esfera personal, y establecen clasificaciones entre los diferentes estímulos afectivos positivos -como el entusiasmo, la felicidad y el cuidado- y negativos -como el miedo, la ira y la tristeza-, que desencadenan determinadas actitudes y comportamientos que pueden beneficiar o perjudicar su buen desempeño, así como las relaciones que establecen con sus compañeros de trabajo y estudiantes. Por su parte, las investigaciones de Kemper (1978) y Lazarus (1999) definen estas sensaciones internas, no siempre conscientes, como un complejo sistema de creencias, motivaciones y significados que los sujetos organizan en función de ciertas vivencias y contextos que modifican sus proyectos de vida.

A pesar de los avances teóricos y metodológicos en el campo, aún se requiere profundizar sobre los rasgos personales que coadyuvan a que algunos académicos presenten una formación de calidad, alcancen altos puntajes en sus evaluaciones y mantengan una participación activa en la investigación. Si bien los estudios sobre el tema enumeran una lista de habilidades -emocionales y cognitivas- que benefician el desarrollo de diferentes actividades intelectuales y sociales, buena parte de sus aportes se concentran en el ejercicio de la docencia, lo que da por hecho que estos caracteres o experiencias sean equiparables a los buenos resultados que obtienen en la investigación; sin advertir que, en ocasiones, entre ambas funciones existen diferentes indicadores o parámetros que miden la eficiencia.

No obstante, en los últimos cinco años se han emprendido estudios que profundizan en las competencias y actitudes que intervienen en la creación de nuevos conocimientos (Sié \& Yakhlef, 2013; Henriksen, 2016). En México, por ejemplo, se ha desarrollado un marco conceptual que permite identificar los indicadores que definen las buenas prácticas en la ciencia a través de conceptos como ética e integridad científica, valores profesionales y responsabilidad social (Hirsch, 2010, 2017). De acuerdo con estos estudios, podemos afirmar que el éxito no se obtiene de una vez y para siempre, pues los investigadores requieren autonomía y un largo proceso de formación, en el que adquieren experiencias significativas que modifican sus conocimientos y los impulsan a elaborar versiones mejoradas de sí mismos para responder a los cambios y las demandas del sector educativo. 


\section{Un modelo para estudiar la excelencia en la investigación}

Los trabajos que analizan los rasgos de la personalidad que influyen en el rendimiento académico retoman con mayor frecuencia el modelo de Eccles y Wigfield (2002), que establece que las motivaciones que los sujetos presentan hacia el desarrollo de una actividad en especial, en este caso la investigación, se relacionan con el valor que le otorgan al trabajo intelectual y con su propio desempeño. Para comprender mejor esta propuesta, los autores establecen tres dimensiones: creencias sobre su competencia y expectativas de éxito, motivaciones, valores, y procesos motivacionales y cognitivos.

En este apartado retomamos dicha propuesta, aunque con algunas adaptaciones que contribuyen a explicar los factores personales que intervienen en la actividad científica, los cuales se agrupan en tres dimensiones (ver esquema 1 ).

Esquema 1. Dimensiones de la excelencia

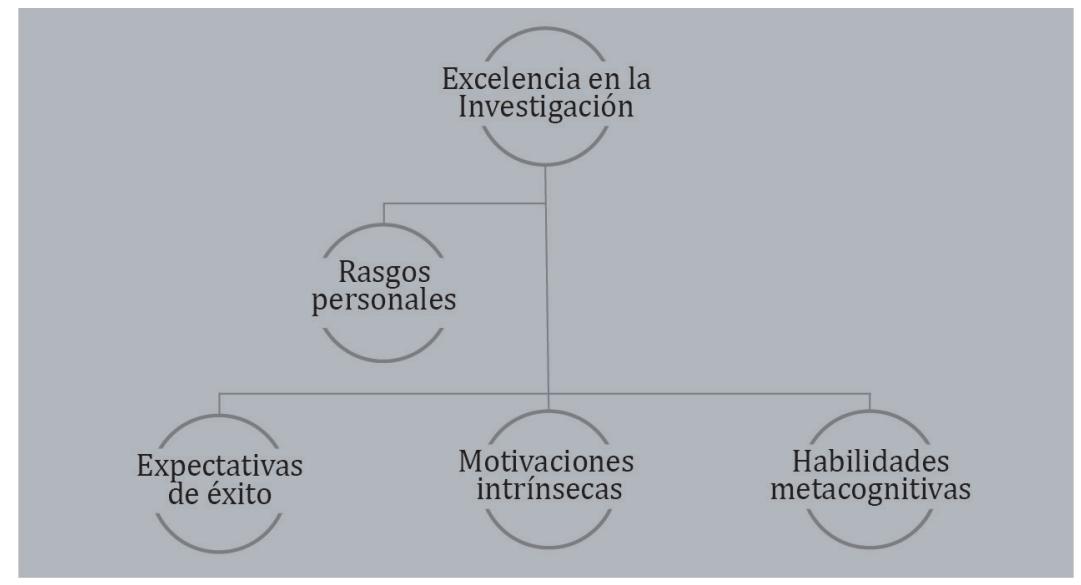

Fuente: elaborado con base en la propuesta de Eccles y Wigfield (2002).

La primera dimensión se encuentra conformada por los rasgos que coadyuvan a que los profesores puedan reconocer y potenciar su talento para la investigación, como autoeficacia, proactividad y autoestima. La segunda se integra por las motivaciones intrínsecas que ocasionan que algunos profesores manifiesten una fuerte inclinación por la ciencia. Estas disposiciones responden a sentimientos e ideales internalizados de manera libre e intuitiva. A diferencia de las motivaciones extrínsecas e instrumentales, como el contexto o los bienes materiales, se caracterizan por el gusto o placer que sienten al leer, escribir e intercambiar ideas.

Uno de los rasgos más relevantes de esta dimensión es la "pasión por la vida intelectual". Si bien su relación con la construcción de nuevos conocimientos no se detalla con claridad, en algunos estudios se aprecian avances importantes respecto a su definición -aunque no precisamente en referencia a la investigación-; a diferencia del "interés" o el "gusto, este rasgo permanece estable en la identidad (Vallerand, 2008) y, a su vez, tiene una gran influencia en el bienestar emocional y la satisfacción laboral (Moè, 2016), al favorecer el trabajo colaborativo y las relaciones interpersonales (Sié \& Yakhlef, 2013).

Finalmente, en la tercera dimensión analizamos la relación que se genera entre las emociones positivas y las habilidades cognitivas durante el ejercicio de la profesión, 
en el cual la motivación y el interés en un determinado tema o tarea benefician la disposición para aprender y crear. En el caso de los científicos, la preferencia por la investigación es el motor que los impulsa a mantenerse constantemente actualizados e involucrarse en diferentes actividades y proyectos académicos (Seery, 2011; Gu \& Day, 2007). El grado de afinidad que han alcanzado con su trabajo y sus aspiraciones personales se ve reflejado en el alto rendimiento que reportan en sus evaluaciones y en la capacidad para adaptarse a los cambios.

\section{Metodología}

Nuestro estudio se apoya en el enfoque comparativo cualitativo propuesto por Ragin (2007) y en el método comparativo constante de Boeije (2002). De acuerdo con estas precisiones, dicho proceso se desarrolló en tres fases: en la primera describimos los casos de manera individual y colectiva; en la segunda, comparamos los relatos por institución y establecemos relaciones de forma cruzada; y en la última analizamos los resultados (ver esquema 2).

Esquema 2. Metodología comparativa cualitativa

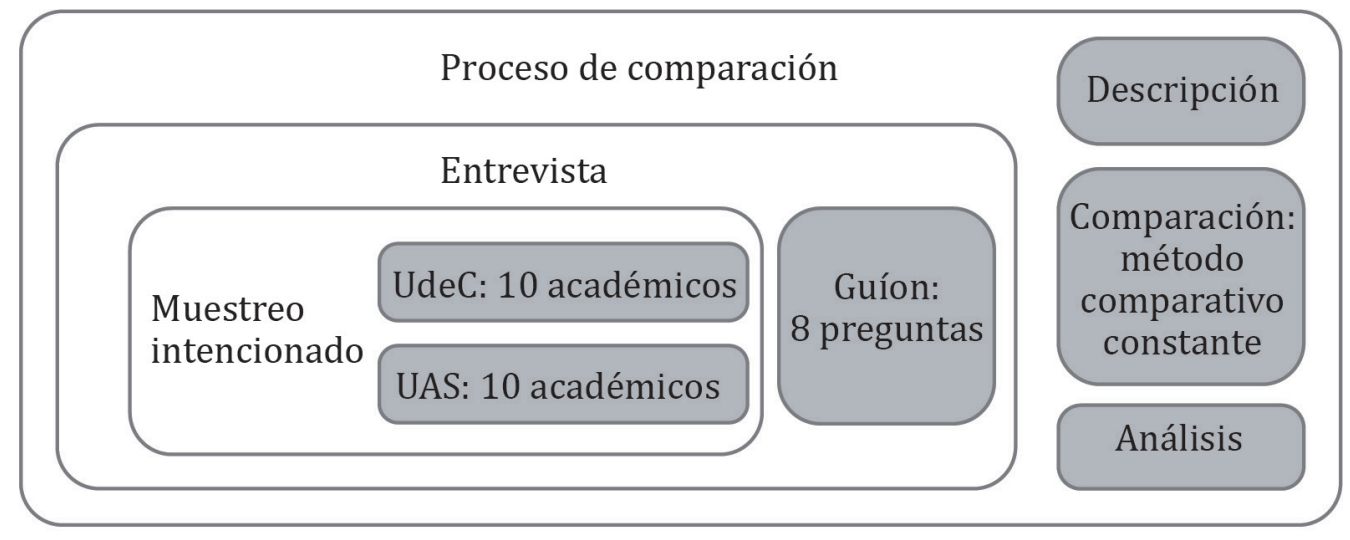

Sujetos de estudio

Uno de los aspectos que caracteriza este enfoque es la sistematicidad en el análisis y la familiarización con los participantes (Ariza y Gandini, 2012). Por ello, coincidimos con Ragin (2007) en la necesidad de seleccionar pocos casos, que no excedan de treinta participantes, de acuerdo con Villalpando (2000). Por esta razón, en cada institución seleccionamos a diez académicos a través de un muestro intencional (McMillan \& Schumacher, 2001) y no de tipo probabilístico, pues no perseguíamos la representatividad estadística, sino conocer en profundidad a los participantes y aprovechar al máximo su formación y experiencia en la investigación.

De este modo, establecimos cinco criterios para su selección: a) que presentaran una contratación de tiempo completo en una de las universidades de estudio; b) pertenecieran al SNI; c) contaran con un "perfil deseable" acorde con los requisitos que plantea el Prodep; d) ostentaran premios y distinciones en el ámbito científico; y e) existiera una equidad de género entre los participantes. Esta información se obtuvo de la página web de cada universidad, del SNI y el Prodep; además, contamos con el apoyo de los asesores pedagógicos y coordinadores de los posgrados de la UdeC y la UAS. 
Tabla 1. Participantes del estudio

\begin{tabular}{|c|c|c|c|}
\hline \multicolumn{2}{|c|}{ Muestra } \\
\hline \multicolumn{2}{|c|}{ Características } & UdeC & UAS \\
\hline \multirow{2}{*}{ Sexo } & Masculino & 4 & 5 \\
\cline { 2 - 4 } & Femenino & 6 & 35 a 62 años \\
\hline \multicolumn{2}{|c|}{ Edades } & 36 a 70 años & 2 a 36 años \\
\hline \multicolumn{2}{|c|}{ Tiempo de servicio } & 6 a 30 años & 5 \\
\hline \multicolumn{2}{|c|}{ Áreas de conocimiento } & Todas, excepto biotecnología y ciencias agropecuarias \\
\hline \multirow{2}{*}{ Miembros del SNI } & I & 7 & 2 \\
\cline { 2 - 4 } & II & 1 & 3 \\
\hline
\end{tabular}

Fuente: elaboración con base en las características de la muestra.

Es preciso resaltar que en ambas universidades elaboramos el conocimiento informado de la investigación mediante un oficio que entregamos a cada académico y director de la facultad o instituto de investigación. En este documento expusimos los objetivos y principales alcances del proyecto, los indicadores que seguimos para la selección de los sujetos de estudio y el compromiso de guardar absoluta reserva de los datos personales de los entrevistados.

\section{Estrategia de investigación}

Para la recolección de la información empírica, aplicamos entrevistas semiestructuradas, entre 2014 y 2015, a través de un guion de ocho preguntas cuyo objetivo era conocer las trayectorias académicas de los participantes; los problemas y dilemas que habían enfrentado a lo largo de su formación profesional e incorporación laboral; las experiencias de tutoría o acompañamiento que impactaron en su identidad; y el modo en que se identificaban con su trabajo en el área científica.

\section{Método y análisis de los resultados}

Ragin (2007) establece tres actividades: descripción, análisis e interpretación de los resultados. Sin embargo, el método que propone no resulta funcional para esta investigación, pues se afianza en instrumentos de corte cuantitativo y se auxilia del algebra booleana para reducir el número de variables a través de la conversión de las proposiciones lógicas en símbolos matemáticos, y realizar operaciones de adición y multiplicación basadas en una lógica combinacional (Ariza y Gandini, 2012). Por este motivo, hicimos algunas adaptaciones al modelo (ver esquema 3 ). 


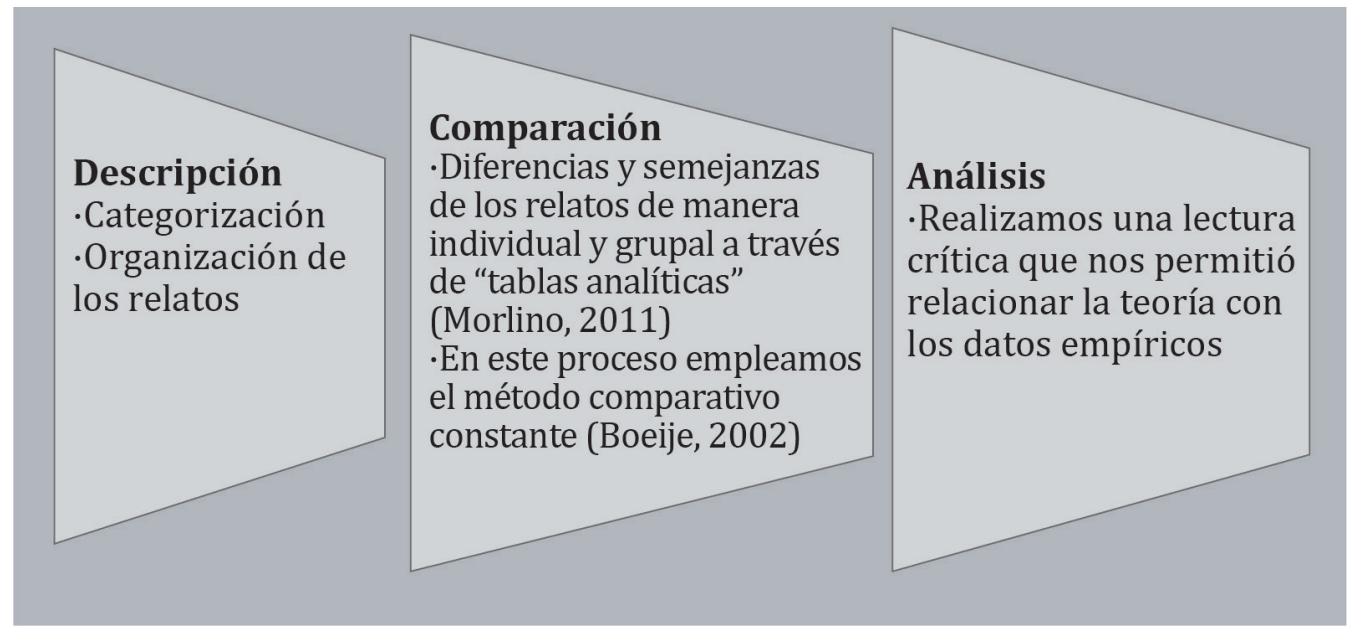

Fuente: elaboración con base en la propuesta de Ragin (2007), Morlino (2011) y Boeije (2002).

En la primera fase, la información recuperada de las entrevistas se codificó en función de las tres dimensiones propuestas en el marco teórico: a) expectativas de éxito: autoeficacia, proactividad y autoestima; b) motivaciones personales: interés o pasión por la investigación; y c) habilidades metacognitivas: estrategias de adaptación y autonomía. En ese proceso obtuvimos una nueva agrupación de las categorías, que organizamos en tres grupos de acuerdo con el orden de importancia y repeticiones: proactividad, pasión por el conocimiento y resiliencia (ver tabla 2).

Tabla 2. Proceso de categorización

\begin{tabular}{|c|c|c|}
\hline Objeto de estudio & Dimensiones & Categorías finales \\
\hline \multirow{2}{*}{$\begin{array}{c}\text { Rasgos personales de } \\
\text { los investigadores }\end{array}$} & Expectativas de éxito & Proactividad \\
\cline { 2 - 3 } & Motivaciones personales & Pasión por el conocimiento \\
\cline { 2 - 3 } & Habilidades metacognitivas & Resiliencia \\
\hline
\end{tabular}

Fuente: elaboración con base en la literatura sobre el tema.

En la segunda fase desarrollamos el proceso de comparación identificando diferencias, semejanzas y relaciones entre los rasgos que distinguen a cada sujeto de manera individual y por institución. Para ello, elaboramos tablas de doble entrada que nos permitieron reconocer qué componentes y eventos provocan que, en una misma categoría, existan opiniones similares y divergentes (Morlino, 2011). En este proceso utilizamos el método comparativo constante de Boeije (2002), que establece una comparación sistemática y de manera cruzada (ver tabla 3).

Tabla 3. Comparación de las entrevistas

\begin{tabular}{|c|c|c|c|c|c|c|c|c|c|c|c|c|c|c|c|c|c|c|c|c|}
\hline Casos & \multicolumn{1}{|c|}{ Universidad de Colima } & \multicolumn{7}{c|}{ Universidad Autónoma de Sinaloa } \\
\hline Entrevistas & $\mathbf{1}$ & $\mathbf{2}$ & $\mathbf{3}$ & $\mathbf{4}$ & $\mathbf{5}$ & $\mathbf{6}$ & $\mathbf{7}$ & $\mathbf{8}$ & $\mathbf{9}$ & $\mathbf{1 0}$ & $\mathbf{1}$ & $\mathbf{2}$ & $\mathbf{3}$ & $\mathbf{4}$ & $\mathbf{5}$ & $\mathbf{6}$ & $\mathbf{7}$ & $\mathbf{8}$ & $\mathbf{9}$ & 10 \\
\hline Categoría 1 & $\uparrow$ & & & & & & & & & & & & & & & & & & \\
\hline Categoría 2 & & & & & & & & & & & & & & & & & & & \\
\hline Categoría 3 & $\downarrow$ & & & & & & & & & & & & & & & & \\
\hline
\end{tabular}

Fuente: elaboración con base en la propuesta de Morlino (2011). 
En la tercera fase llevamos a cabo dos procedimientos: la metasíntesis y el análisis final de los resultados. En la primera actividad buscamos reducir la información que obtuvimos de la comparación a través de una lectura crítica que nos permitió eliminar elementos que no guardaban relación con el objetivo de la investigación. Finalmente, realizamos la relación de los datos empíricos con la teoría, ejercicio que coadyuvó al análisis final.

\section{RASGOS PERSONALES DE LOS INVESTIGADORES EXITOSOS DE LA UDEC Y LA UAS}

Los autores que abordan las cualidades personales de los profesores competentes destacan su papel activo en la conformación de situaciones de aprendizaje que potencien la búsqueda de nuevos conocimientos, al planear interacciones productivas, que permitan afrontar con éxito las exigencias del ámbito académico. En el caso de los entrevistados, es preciso mencionar que a lo largo de sus trayectorias han adoptado el rol de aprendices o noveles en el ejercicio de la profesión. Así pues, producto de estas fases de inducción y nuevos retos, han conformado un conjunto de capacidades y actitudes que contribuyeron a que culminaran su carrera, accedieran a una plaza de tiempo completo e incursionaran en la actividad científica.

A pesar de que en este proceso existen diferencias individuales, buena parte de estos resultados se deben a la presencia de tres rasgos en común: conducta proactiva, pasión por el conocimiento y resiliencia.

\section{Conducta proactiva}

Cuando una persona toma el control de su propio proceso formativo y planifica un conjunto de acciones para resolver posibles conflictos o superar los obstáculos institucionales, se habla de una conducta proactiva. Este concepto está estrechamente relacionado con el de autoformación, propuesto por Dumazedier (1998) y adaptado por Yurén (2005) para explicar el desarrollo profesional de los docentes. En ambos casos, la toma de poder inicia cuando las expectativas son puestas en cuestionamiento y las formas de educación tradicional no satisfacen las necesidades intelectuales de los sujetos, lo que motiva el diseño o búsqueda de nuevas opciones o situaciones de aprendizaje. Como el caso de una profesora de la UAS que decidió a la mitad de la carrera de Psicología matricularse en el Sistema de Educación Abierta con la intención de tener más tiempo para participar en un proyecto de investigación:

Trabajé en una encuesta de investigación muy seria, auspiciada por la ONU [...]. Yo tenía veinte años, andar $p a^{\prime}$ arriba, $p a^{\prime}$ abajo encuestando. Me pasé a la universidad abierta, para culminar la carrera [...]. Entonces, perdí la cuestión esa de amistad [...], pero pues la gané en otro sentido no ¿verdad? (entrevista 5, UAS, 12/2/15).

La capacidad de tomar las riendas de su formación y reflexionar sobre su futuro laboral ha motivado a que gran parte de los entrevistados exploren nuevos espacios educativos que los acerquen a su ideal profesional, al modificar o renunciar a ciertos enfoques epistemológicos y transitar por diferentes disciplinas. Como se aprecia en el relato de un investigador de la UAS, que estudió en una escuela normal y que el deseo de especializarse en el estudio de la lingüística lo llevó a cursar un posgrado en la universidad: 
Los que somos maestros normalistas sabemos que [...] el sistema de las normales es rígido [...] para incursionar en temáticas distintas [...], sobre todo en la investigación [...], y de cuarto y quinto que estaba en la normal, tomé la decisión de cambiarme a la lingüística y dejar a la literatura en paz [...]. Entonces, [...] me fui a la UNAM a estudiar la maestría en lingüística [...], y luego me integré como asistente de investigación (entrevista 2 , UAS, 9/2/15).

Al no encontrar los cursos o programas que compensaran sus intereses intelectuales, algunos profesores desarrollaron de manera creativa y autodidacta medios y recursos cognitivos que los aproximara al conocimiento. El caso de un profesor de la UAS sirve de ejemplo, pues su interés prematuro por la "relatividad" lo impulsó a buscar información en libros y folletos; este ejercicio fortaleció su independencia y el talento para afrontar posteriores dilemas:

Yo ya en la secundaria me distinguía porque tenía facilidades en física y matemáticas. Entonces, un profesor [...] me regalo un libro de la relatividad [...] y desde tercero de secundaria decidí que iba a incursionar en ese tema [...]. Mi problema fue que al entrar en la prepa [...], y luego en los primeros años de la universidad, tampoco recibe cursos de relatividad. Entonces, [...] lo tuve que hacer por mi cuenta. Entonces, me compré unos libros muy abstractos [...] y poco a poco lo fui abordando (entrevista 1, UAS, 9/2/15).

La facilidad para desarrollar estrategias que los ayudaran a resolver posibles conflictos y adaptarse a contextos desafiante no solo dependió de su buena disposición para aprender, sino también de su madurez y un bagaje cultural que los ayudó a enfrentar las nuevas exigencias educativas. El caso de una académica de la UdeC resulta interesante porque describe cómo el deseo de mejorar la pronunciación del inglés, mientras realizaba un doctorado en Boston, la condujo a idear una serie de medidas poco convencionales:

Yo ya sabía el idioma [inglés], entonces eso no fue un problema, quizás es diferente la pronunciación y hay ciertas palabras diferentes del británico [...], pero lo que hice fue irme a misa, me sentaba todo el día para estarlos escuchando o veía películas y la adaptación fue más rápida en cuanto a los acentos, pero de ahí en más no hubo problema (entrevista 6, UdeC, 28/11/14).

Estos ejemplos no solo forman parte de sus anécdotas estudiantiles, sino que con el paso de los años adquirieron mayor formalidad y notoriedad en su personalidad y se transformaron en estilos y maneras de asumir su trabajo en la universidad y dirigir su vida. El relato de un entrevistado de la UdeC describe mejor esta idea, pues la preocupación por incorporar nuevos elementos teóricos a su proyecto sobre el flujo sanguíneo influyó en el deseo de realizar un año sabático en Londres:

Me veo como una persona que siempre está en desarrollo y queriendo descubrir cosas nuevas, ahora lo he visto más con esta parte del año sabático [...], aquí la gente no entiende que me quiera ir [...], soy la primera persona [...] que se va [...], y la verdad es que lo veo como una necesidad muy personal de ver cosas nuevas, o sea no me podría quedar nada más así [...], siempre trato de conocer cosas nuevas que me impacten (entrevista 1, UdeC, 17/11/14).

Es preciso aclarar que, a pesar de que los entrevistados presentan trayectorias caracterizadas por su entrega y compromiso con su trabajo, no en todas las fases de su carrera se aprecia una conducta proactiva. Sin embargo, la mayoría de ellos desarrollan "mecanismos o tácticas de agencia" para negociar, adaptar o reformar las prácticas y normas impuestas por sus universidades y regímenes externos, como 
el SNI o el Prodep. Por esta razón, consideramos que sus biografías se construyen y reconstruyen en función de las restricciones, los conflictos y las divergencias que enfrentan a lo largo de su formación.

Si bien las instituciones universitarias y el capital cultural tienen una fuerte influencia en su formación. Las experiencias expuestas en este apartado demuestran que los rasgos personales -inteligencia, ingenio y autonomía- cumplen también un papel importante en la búsqueda de nuevas oportunidades de desarrollo, así como en el fortalecimiento de la reflexión, la eficacia y el vínculo con otros agentes institucionales.

\section{Pasión por el conocimiento}

Se define como aquel gusto o fuerte inclinación que sienten los profesores por adquirir nuevos conocimientos, que los motiva a dedicar gran parte de su tiempo y energía a realizar diferentes actividades intelectuales que coadyuven a satisfacer sus necesidades personales y fortalecer sus competencias profesionales. A medida que su práctica logra ocupar un lugar significativo en sus vidas, se convierte en una característica central de su identidad y tiene una gran influencia en su vocación por la investigación (Vallerand et al., 2003; Quijada, 2017).

Por ello, en sus relatos es visible el deseo de mantenerse actualizados, dominar ciertos temas, competir con sus pares y explorar nuevos espacios académicos y culturales que contribuyan a que puedan alcanzar sus aspiraciones profesionales. Así lo describe una investigadora extranjera que trabaja en la UdeC y cuyo interés por especializarse en el estudio de poblaciones vulnerables ayudó a que realizara un doctorado en Migración:

En toda la licenciatura yo trabajaba en una organización que se llama Habitat for Humanity [...] que construye casas [...], como era un área que no se había estudiado tanto [...], pues ya apliqué y me llevó a la migración [...], en el doctorado yo quedé con una familia de Chihuahua y ya empecé a leer... (entrevista 3, UdeC, 19/11/14).

Sin embargo, algunos profesores concuerdan en que el anhelo de aprender no fue suficiente, pues en ocasiones se requiere sistematicidad y disciplina para enfrentar las exigencias del posgrado e incorporar los consejos de sus asesores. Al respecto, una entrevistada de la UdeC que pertenece al área de historia menciona que, a medida que asumió nuevos desafíos educativos, fue desarrollando un conjunto de estrategias cognitivas y emocionales que coadyuvaron a que culminara sus estudios de doctorado en París:

Cuando eres inquieta y tienes aspiraciones y quieres seguir formándote, pues vas subiendo escaloncitos [...]. Yo me di cuenta de esto muchos años después, [...] estando en París, una tarde tenía ya como un mes y estaba haciendo un proyecto [...], me la había pasado en la Biblioteca Nacional de Francia todo el día, había comido un sándwich en el baño por seguir trabajando [...]. Yo misma me la construí, mi forma de destacar (entrevista 2 , UdeC, 25/11/14).

Los desvelos, las largas jornadas de estudio y su perseverancia, entre otras experiencias, describen a profesionales con un alto grado de responsabilidad y compromiso, cuyo esfuerzo se encuentra materializado en sus tesis y su producción científica, así como en los diferentes procesos de formación que han atravesado para lograr un 
estilo propio y cierto reconocimiento. El comentario de una profesora que labora en la Facultad de Economía de la UdeC sirve de ejemplo:

Siempre quise ser excelente y siempre busco serlo, es parte de mí ser perfeccionista, no estoy conforme conmigo misma, si no hago las cosas bien [...]. Al principio mis publicaciones fueron hacia cuestiones más que nada nacionales [...], pero ya con el tiempo empecé a abrirme camino [...], tengo audiencia [...], pero hay que saberte organizar [...], balancear tu tiempo (entrevista 6, UdeC, 25/11/14).

Otra de las cualidades que se advierte en la manera en que perciben su trabajo y desarrollan sus investigaciones es el interés por determinados temas y proyectos de investigación, que los han llevado a introducirse en diferentes disciplinas o, como lo narra un profesor que pertenece a la Facultad de Física de la UAS, a reformular sus enfoques o posturas teóricas:

En 1984 inicia la primera revolución de súper cuerdas y entonces eso influyó mucho [...], me empecé a preocupar en proponer mis propias teorías [...] y a partir de ahí fui participando junto con investigadores internacionales [...]. Ahora, con más libertad, más consolidado, pues he estado buscando teorías de gravitación [...], con la idea de incursionar o tratando de ver qué está haciendo falta (entrevista 1, UAS, 9/2/15).

El ímpetu de ser cada día mejor, superar sus carencias y traspasar sus propios límites intelectuales, se nutre de esa satisfacción que sienten cuando hacen lo que más les gusta, renueva y fortalece. De ahí que el éxito no solo demanda rigor y dedicación, sino, sobre todo, pasión y vocación, como describe una investigadora que labora en la Facultad de Medicina de la UAS:

Para mí es casarte con una carrera, tenerla que amar, poderte casar para poderla transmitir y poder aguantar muchas cosas. Porque si no la amas, vas hacer las cosas por hacer o no las vas hacer y te quedas sin avanzar, porque esto requiere mucho tiempo, requiere mucho esfuerzo, dejas de lado muchas cosas, familia [...], por estar haciendo tu trabajo de campo, eso es lo que implica ser investigador (entrevista 8, UAS, 17/2/15).

Sin bien existen diferencias sustantivas respecto a la pasión que sienten por generar e intercambiar nuevos conocimientos, pues en algunos casos se advierte con mayor fuerza que en otros, la mayoría coincide en que el nivel de satisfacción que han alcanzado con su trabajo ha repercutido de manera significativa en su interés por la investigación. Sin embargo, con el paso de los años esta pasión se ha modificado debido al control que ejercen las universidades y los sistemas de evaluación sobre su trabajo, ya que con frecuencia los enfrentan a diferentes conflictos y dilemas para tratar de conjugar sus motivaciones personales con las expectativas institucionales. Esta situación, en ocasiones, desencadena problemas emocionales y actitudinales relacionados con el estrés, el cansancio y la desilusión. Esto se aprecia en el testimonio de un académico que cuenta con el nivel más alto en el SNI:

Ya tengo como veintisiete años en el Sistema Nacional de Investigadores. Hay momentos en que sí se cuestiona uno, ¿no?, porque dice uno, estoy trabajando para Conacyt, para la universidad o para desarrollar mis propias ideas. Muchas veces dice uno, pues si me subieran el salario. Este, pues tendría más libertad, este haría cosas más personales (entrevista 1 , UAS, 9/2/15).

A pesar de estas dificultades y cuestionamientos, su tenacidad no se ha quebrantado, sino todo lo contrario, han aprendido a modificar o reformular sus expectativas y proyectos de vida sin perder de vista el motor que dirige buena parte de sus 
trayectorias, como es la pasión por el conocimiento, rasgo que ha caracterizado su actuación y sus metas desde su etapa estudiantil.

\section{Resiliencia}

De acuerdo con Cyrulnik (2003), se define como la capacidad que presentan los seres humanos para soportar o revertir las situaciones adversas a través de la reorganización de las emociones, actitudes y percepciones. Para ello se apoyan en las experiencias (positivas y negativas) pasadas y en los vínculos que han establecido con los diferentes agentes sociales e institucionales. Sobre este punto, Seery (2011) destaca que, si bien existen eventos que traen consecuencias perjudiciales para la salud física y psicológica, su aprendizaje resulta importante para alcanzar el éxito en la vida, pues fortalecen la autoestima, el control y la adaptación a diversos escenarios.

En el ámbito académico, la resiliencia es un tema de investigación emergente que ha cobrado relevancia en los últimos años (Burns, Poikkeus \& Aro, 2013), y se define como aquella habilidad o pericia que adquieren los profesores al introducirse en contexto y dinámicas desafiantes que ponen en riesgo su estabilidad laboral y bienestar personal (Castro, Kelly \& Shih, 2010); las más recurrentes son el estrés, las enfermedades y la reducción del salario. Para afrontar estos periodos de cambio o conflicto, crean y gestionan diversos mecanismos de defensa que, de acuerdo con Gu y Day (2007), se agrupan en dos bloques: la formación de redes de apoyo y el fortalecimiento de los atributos psicológicos.

El primer componente se refiere al entorno social o grupo de personas que los protegen y socorren durante circunstancias adversas. En el caso de los sujetos de este estudio, destacan la asistencia y el soporte emocional que recibieron de parte de algunos de sus profesores. La experiencia de una entrevistada sirve de ejemplo, al describir cómo superó las dificultades económicas que impedían que continuara sus estudios de maestría con el respaldo y apoyo moral de su asesor:

Cuando yo entré a la maestría había un déficit en Conacyt por problemas de que bajó el petróleo en Pemex, entonces, estaban dando una beca incompleta y él [asesor] me ayudó a tramitar [...] una beca complementaria porque yo tenía un hijo. Entonces, a mí se me hizo muy difícil. Entonces, él me dijo, no se preocupe vamos a buscar la manera y me ayudó (entrevista 8, UdeC, 2/14/14).

La colaboración y atención de sus amigos o colegas de trabajo en momentos difíciles también fue un aliciente que les proporcionó seguridad y motivación para continuar con sus planes a través de la solidaridad, la cohesión y la comprensión. Así se advierte en la narración de una investigadora de la UdeC, quien señala que la fraternidad que existe entre sus compañeras de cuerpo académico le ayudó a enfrentar algunos problemas de tipo personal:

En los últimos años el trabajo con las colegas del cuerpo académico, eso ha sido muy importante porque cada una de nosotras ha pasado por etapas difíciles, hemos tenido, por ejemplo, una compañera que recientemente ya va saliendo de una enfermedad complicada, por ejemplo, otra compañera que también enfrentó un problema familiar muy severo [...], yo también he tenido mis altibajos y el trabajo colaborativo o la participación en conjunto, te da muchos ánimos, te ayuda (entrevista 10, UdeC, 5/12/14). 
La intervención de instituciones y organizaciones como el Conacyt, el Prodep y Relaciones Exteriores, entre otras entidades, también ha sido un apoyo determinante para que puedan concluir su formación científica; esto, a través de subsidios o financiamientos educativos. Así lo indica un investigador de la UAS que pudo culminar sus estudios de posgrado gracias a una beca:

Yo no tenía muchos recursos económicos [...]. Ante la falta de trabajo [...], me fui a Estados Unidos [...] estuve allá trabajando de brasero. Regresé porque me echó la migra [...] [se ríe]. Cuando llego a Culiacán, leo en el periódico que estaba la opción de estudiar la maestría [...], prometían muchas cosas ahí, de que sí tú pasabas los exámenes ibas a estudiar y ¡becado!, así fue como estudié la maestría (entrevista 9, UAS, 17/2/15).

A lo largo de sus trayectorias el soporte de diferentes agentes ha jugado un papel importante en su desarrollo académico, al igual que su entrega y entereza para buscar ayuda y responder de forma asertiva a los obstáculos. De ahí que el segundo factor propuesto por Gu y Day (2007) se relaciona con la autoestima y la eficacia, es decir, con aquella carga emotiva que impacta en su componente de valores y fortalece su vocación, la satisfacción laboral y los deseos de superación. Así lo asevera un profesor de la UAS, cuyo deseo por adquirir nuevos conocimientos le permitió culminar un posgrado en Polonia:

El doctorado era una opción natural, la institución impulsaba y yo tenía, más o menos, ciertas metas ¿no?, características de superación personal [...]. Me fue un poco para mí [...], al principio, un poco dramático, como era en el extranjero, tenía que separarme tiempo de mi familia [...], pero fue satisfactorio [...], hice bien en aceptar (entrevista 3, UAS, 10/2/15).

La internalización de estas experiencias contribuyó al desarrollo del pensamiento positivo que, en buena parte de los casos, guiaron su reinvención y empoderamiento profesional. Sin embargo, como lo señala Seery (2011), en ocasiones los eventos negativos se convirtieron en el estímulo que los obligó a reconfigurar sus motivaciones. La experiencia de un profesor de la UdeC que encontró en el aprendizaje del idioma ruso un medio de motivación y sostén para continuar con sus estudios de doctorado en un país que atravesaba una crisis política ilustra mejor esta idea:

Yo entré a la Unión Soviética [para estudiar el posgrado], entré a un país y egresé de otro [...], fueron años muy complicados [...], yo ya me quería regresar, fue impactante el choque cultural, pasados los cuatro meses logré superar eso y una salida que tuve fue el aprendizaje del idioma ruso, porque me dediqué prácticamente catorce horas diarias a estudiarlo (entrevista 4, UdeC, 28/11/14).

En las dos universidades existen ejemplos que demuestran que es posible superar las limitaciones personales y del contexto, pues sus trayectorias retratan una lucha continua por alcanzar su consolidación como investigadores. Un punto que resaltan es el aporte de los distintos programas de becas, así como el apoyo de algunos profesores y familiares, además de su propio esfuerzo y el deseo de mejorar su estatus social. Sin embargo, también se identifican factores de riesgo que frustran sus metas y planes, entre los que destacan el déficit económico, los ambientes académicos hostiles, las crisis familiares y los problemas de salud. 


\section{DisCUSIÓN DE LOS RESULTADOS}

El estudio de los rasgos personales que caracterizan a los profesores exitosos en la UdeC y la UAS coadyuvó a establecer una ruta común en su formación científica, en la cual las enseñanzas y experiencias significativas que adquirieron en la universidad -principalmente en el posgrado- fortalecieron su inclinación por la ciencia y sus habilidades metacognitivas. En esta etapa, el acompañamiento de buenos profesores, la obtención de becas de posgrado y las estancias en el extranjero influyeron en su buen rendimiento académico. Sin embargo, estos resultados no hubiesen sido posibles sin las altas expectativas que presentaban de sí mismos y de su carrera, que los impulsó a planificar y potenciar sus conocimientos de manera proactiva, incluso a pesar de las dificultades.

Las diferencias más significativas en sus trayectorias se encuentran relacionadas con la edad y las políticas educativas. Los profesores que estudiaron entre la década de los setenta y ochenta tardaron de uno a cinco años para empezar una maestría o doctorado, y algunos tuvieron problemas económicos para continuar sus estudios, pues en este periodo aún no se oficializaban las becas o subsidios para la investigación. En contraste, quienes iniciaron un posgrado a mediados de los noventa y en adelante presentaron menos interrupciones y culminaron su doctorado a una edad no mayor de treinta y cinco años. Además, afrontaron menos dificultades para acceder a financiamientos e ingresar a universidades fuera del país, ya que algunos dominaban una segunda lengua.

Respecto a la motivación intrínseca por la academia, también advertimos discrepancias, pues los académicos que se formaron antes de la creación del SNI y el Prodep reafirmaron su interés en la investigación al estudiar un doctorado, incluso algunos ya contaban con una plaza como profesores de asignatura o de tiempo completo. En contraste, los más jóvenes comenzaron a participar en proyectos de investigación desde la licenciatura o maestría y, en ese periodo, más de la mitad ya habían publicado un artículo o capítulo de libro.

Uno de los principales factores que no benefician el desarrollo de la ciencia en ambas universidades es la escasa creación de posgrado de calidad. Por esta razón, solo dos profesores estudiaron una maestría en la UdeC y los demás, al igual que en la UAS, se formaron en la Ciudad de México o en el extranjero. En el plano personal, la mayoría concuerda que en algún momento de su carrera experimentó problemas económicos o familiares, como el escaso tiempo para el cuidado de los hijos o las rupturas sentimentales. A pesar de que estos eventos les generaron estrés y emociones negativas, su pasión por la investigación no se debilitó e, incluso, en algunos casos fue un aliciente que disipó o atenuó sus conflictos.

Durante su formación y desempeño profesional, advertimos la presencia de una conducta proactiva, pues se auxilian de libros, cursos y congresos que seleccionan de acuerdo con sus intereses intelectuales. Sin embargo, algunos de estos aprendizajes son impuestos por sus universidades, en las que deben asumir diferentes roles, que requieren su experiencia en el ámbito de la docencia y la gestión. Por ello, en el plano laboral se agencian de espacios de microautonomía que les ayuden a recuperar la dirección de su carrera y sus motivaciones personales. De ahí que el buen ejercicio de sus funciones no solo se encuentra condicionado al cumplimiento de ciertos parámetros institucionales, sino que depende de su madurez emocional y cognitiva para administrar su tiempo y esfuerzo de manera acertada. 
Si bien los rasgos personales que han influido en el éxito de los investigadores de la UdeC y la UAS se exponen por separado, en la práctica resulta difícil su división, ya que están estrechamente relacionados y funcionan en forma coordinada en la actuación. No obstante, su análisis individual permitió identificar diferencias significativas en su apropiación, pues la pasión por el conocimiento es el elemento que se mantiene más estable en sus biografías y dirige buena parte de sus decisiones y proyectos académicos. En contraste, la proactividad tiene picos de declive a mitad de la carrera y poco antes de la jubilación. Así pues, la resiliencia depende del grado de pasión y proactividad que manifiestan en su carrera, aunque no son los únicos factores que condicionan su formación. Por ello, al igual que otros autores, consideramos que es un constructo multidimensional que adquiere protagonismo en los eventos de cambio o transición.

La conceptualización de estos rasgos personales, así como su adaptación al contexto de las universidades estatales, sirve de referencia a posteriores investigaciones que muestran interés en el tema y, a su vez, abre una línea de análisis para el estudio del éxito profesional en México. Asimismo, permite visibilizar las trayectorias de los investigadores que trabajan en los estados de Colima y Sinaloa, donde, a pesar de las limitaciones institucionales y los problemas económicos, realizan esfuerzos extraordinarios por impulsar el desarrollo científico y tecnológico de sus regiones.

\section{REFERENCIAS BIBLIOGRÁFICAS}

Ariza, M. y Gandini, L. (2012). El análisis comparativo cualitativo como estrategia metodológica. En L. Velasco y M. Ariza (coords.). Métodos cualitativos y su aplicación empírica: por los caminos de la investigación sobre migración internacional (pp. 497-533). México: Universidad Nacional Autónoma de México.

ANUIES (2017, diciembre). Información estadística de educación superior. Recuperado de Anuarios Estadísticos de Educación Superior: http://www. anuies.mx/informacion-y-servicios/informacion-estadistica-de-educacionsuperior

Badia, A., Meneses, J. y Monereo, C. (2014). Affective dimension of university professors about their teaching: An exploration through the semantic differential technique. Universitas Psychological, vol. 13, núm. 1, pp. 161-173. Recuperado de http://ucsj.redalyc.org/articulo.oa?id=64730432014

Bain, K. (2012). Lo que hacen los mejores estudiantes de universidad. Valencia: Universitat de València.

Bain, K. (2004). Lo que hacen los mejores profesores universitarios. Valencia: Universitat de València.

Beijaard, D, Verloop, N. \& Vermunt, J. D. (2000). Teachers' perceptions of professional identity: An exploratory study from a personal knowledge perspective. Teaching and Teacher Education, núm. 16, pp. 749-764. Recuperado de http://www.sciencedirect.com/science/article/pii/S0742051X00000238

Beijaard, D., Meijer, P. \& Verloop, N. (2004). Reconsidering research on teachers' professional identity. Teaching and Teacher Education, núm. 20, pp. 107-128. Recuperado de https://openaccess.leidenuniv.nl/bitstream/handle/1887/11190/10_404_07.pdf?seque 
Boeije, H. (2002). A purposeful approach to the constant comparative method in the analysis of qualitative interviews. Quality \& Quantity, vol. 36, pp. 391-409. Recuperado de http://link.springer.com/article/10.1023 /A:1020909529486

Burns E., Poikkeus, A. \& Aro, M. (2013). Resilience strategies employed by teachers with dyslexia working at tertiary education. Teaching and Teacher Education, núm. 34, pp. 77-85. Recuperado de http://www.sciencedirect.com/ science/article/pii/S0742051X13000747

Canrinus, E., Helms-Lorenza, M., Beijaard, D., Buitinka, J. \& Hofmana, A. (2011). Profiling teachers' sense of professional identity. Educational Studies, vol. 37, núm. 5, pp. 593-608. Recuperado de http://www.tandfonline.com/doi/abs/ $10.1080 / 03055698.2010 .539857$

Castro A., Kelly J. \& Shih, M. (2010). Resilience strategies for new teachers in high needs áreas. Teaching and Teacher Education, núm. 26, pp. 622-629. Recuperado de http://www.sciencedirect.com/science/article/pii/S0742051X09001905

Cyrulnik, B. (2003). Cuando uno está muerto y surge el oculto tiempo de los recuerdos. En B. Cyrulnik. Los patitos feos. La resiliencia: una infancia infeliz no determina la vida (pp. 20-23). Barcelona: Gedisa.

Day, C. (2006). Pasión por enseñar: la identidad personal y profesional del docente y sus valores. Madrid: Narcea.

Day, C. y Gu, Q. (2012). Las condiciones del éxito. En C. Day y Q. Gu (coords.). Profesores: vidas nuevas, verdades antiguas. Una Influencia decisiva en la vida de los alumnos (pp. 149-220). Madrid: Narcea.

Day, C., Kington, A., Stobart, G. \& Sammons, P. (2006). The personal and professional selves of teachers: stable and unstable identities. British Journal of Educational Research, vol. 32, núm. 4, pp. 601-616. Recuperado de http:// onlinelibrary.wiley.com/doi/10.1080/01411920600775316/abstract

Dumazedier, J. (1998). La montée de l'autoformation dans l'éducation permanente: une aproche sociologique et historique. París: Les six thémes, Biennale de l' education et de la formation.

Eccles, J. \& Wigfield, A. (2002). Motivational beliefs, values, and goals. Annual Review of Psychology, vol. 53, núm. 1, pp. 109-132. Recuperado de https:// www.ncbi.nlm.nih.gov/pubmed/11752481

Galaz, J. F. (2002). La satisfacción laboral de los académicos en una universidad pública estatal. Perfiles Educativos, vol. XXIV, núm. 96, pp. 4772. Recuperado de http://www.iisue.unam.mx/perfiles/busqueda. php?indice $=$ autor\&busqueda=GALAZ $\% 20$ FONTES,\%20JESUS $\% 20$ FRANCISCO

Galaz, J. F., Gil, A., Padilla, L., García, J., Martínez, J. y Arcos, J. (2012). La educación superior mexicana en la encrucijada: temas para una agenda en política pública. En J. F. Galaz, M. Gil, L. Padilla, J. García, J. Martínez y J. Arcos (coords.). La reconfiguración de la profesión académica en México (pp. 33-42). México: Universidad Autónoma de Sinaloa/Universidad Autónoma de Baja California.

García-Romero, A. (2012). Influencia de la carrera investigadora en la productividad e impacto de los investigadores españoles. El papel de la ventaja acumulativa. Revista Española de Documentación Científica, vol. 35, núm. 1, pp. 38-60. Recuperado de http://redc.revistas.csic.es/index.php/redc/article/ viewArticle/723 
Góngora, E. (2012). Profesión y carrera académica: referentes para el análisis del prestigio. En E. Góngora (coord.). Prestigio académico: estructuras, estrategias y concepciones (pp. 49-84). México: ANUIES.

Gu, Q. \& Day, C. (2007). Teachers' resilience: A necessary condition for effectiveness. Teaching and Teacher Education, núm. 23, pp. 1302-1316. Recuperado de http://www.sciencedirect.com/science/article/pii/S0742051X06001028

Henriksen, D. (2016). The seven transdisciplinary habits of mind of creative teachers: An exploratory study of award winning teachers. Journal Thinking Skills and Creativity, vol. 22, pp. 212-232. Recuperado de http://www.sciencedirect.com/science/article/pii/S1871187116301444?via\%3Dihub

Hirsch, A. (2017, noviembre). Ética profesional y excelencia del profesorado. En M. A. Buendía (presidencia). Educación y Valores. Simposio llevado a cabo en el XIV Congreso Nacional de Investigación Educativa, San Luis Potosí, México.

Hirsch, A. (2010). Ética profesional y profesores universitarios: una perspectiva comparativa. Revista Reencuentro, núm. 57, pp. 34-38. Recuperado de http:// www.redalyc.org/pdf/340/34012514005.pdf

Hirsch, A. (2008). Valores básicos que la universidad debiera promover en su alumnado y en su profesorado. Profesores del posgrado de la UNAM. En A. Hirsch y R. López (coords.). Ética profesional y posgrado en México (pp. 19-34). México: UNAM.

Kemper, T. D. (1978). A social interactional theory of emotions. Nueva York: John Wiley.

Lazarus, R. S. (1999). Stress and emotion: A new synthesis. Nueva York: Springer.

Martínez- Navarro, E. (2011). Actitudes éticas del profesor en relación consigo mismo, con el contexto ético-político y sus alumnos. En E. Martínez-Navarro (coord.). Ética profesional de los profesores (pp. 131-160). Bilbao: Desclee de Brouwer.

McMillan, J. H. \& Schumacher, S. (2001). Research in education: A conceptual introduction (quinta edición). Nueva York: Longman.

Moè, A. (2016). Harmonious passion and its relationship with teacher well-being. Teaching and Teacher Educationvol, núm. 59, pp. 431-437. Recuperado de https://www.sciencedirect.com/science/article/pii/S0742051X16301457 [Consulta: 12 de enero de 2018].

Morlino, L. (2011). Cómo comparar: los mecanismos esenciales. En L. Morlino (cood.). Introducción a la investigación comparada (pp. 81-118). México: Alianza Editorial.

Quijada Lovatón, K. Y. (2017). La formación de la identidad profesional de los académicos de la Universidad de Colima y la Universidad Autónoma de Sinaloa. Un análisis comparativo. Tesis de doctorado. Universidad Nacional Autónoma de México.

Ragin, C. (2007). El uso de los métodos comparativos para estudiar la diversidad. En C. Ragin (coord.). La construcción de la investigación social. Introducción a los métodos y su diversidad (pp. 177-212). Colombia: Universidad de los Andes.

Seery, M. (2011). Resilience: A silver lining to experiencing adverse life events? Psychological Science, vol. 20, núm. 6, pp. 390-394. Recuperado de http://journals.sagepub.com/doi/abs/10.1177/0963721411424740?journalCode=cdpa

Sié, L. \& Yakhlef, A. (2013). The passion for knowledge: Implications for its transfer. Knowledge and Process Managemen, vol. 20, núm. 1, pp. 12-20. Recuperado de https://onlinelibrary.wiley.com/doi/abs/10.1002/kpm.1402 
Skaalvik, E. M. \& Skaalvik, S. (2009). Does school context matter? Relations with teacher burnout and job satisfaction. Teaching and Teacher Education, vol. 25, pp. 518-524. Recuperado de https://www.sciencedirect.com/science/article/pii/S0742051X08002163

Vallerand, R. (2008). On the psychology of passion: In search of what makes people's lives most worth living. Canadian Psychology, vol. 49, núm. 1, pp. 1-13. Recuperado de https://selfdeterminationtheory.org/SDT/documents/2008_ Vallerand_CanPsych.pdf

Vallerand, R., Mageau, G., Ratelle, C., Le'onard, M., Blanchard, C., Koestner, R., Gagne, M. \& Marsolais, J. (2003). Les passions de l'A^ me: On obsessive and harmonious passion. Journal of Personality and Social Psychology, vol. 85, núm. 4, pp. 756-767. Recuperado de http://psycnet.apa.org/index.cfm?fa=buy. optionToBuy\&id=2003-08110-016

Villalpando, J. M. (2000). La técnica de la comparación pedagógica. En J. M. Villalpando (coord.). Pedagogía comparada, teoría y práctica (pp. 107-124). México: Porrúa.

Visser-Wijnveen, G., Stes, A. \& Petegem, P. (2014). Clustering teachers' motivations for teaching. Teaching in Higher Education, vol. 19, núm. 6, pp. 644-656. Recuperado de https://www.tandfonline.com/doi/abs/10.1080/13562517. 2014.901953

Universidad Autónoma de Sinaloa (2017). Informe de labores 2016-2017. Secretaria Académica Universitaria. Recuperado de http://sau.uas.edu.mx/

Universidad Autónoma de Sinaloa (2012). Dirección de Comunicación Social. Recuperado de http://dcs.uas.edu.mx/index.php?sec=3\&op=2\&tipo=i\&id_ noticia $=2430$

Universidad de Colima (2018). Sistema Institucional de Indicadores. Recuperado de programas educativos: http://planeacion.ucol.mx/indicadoresinstitucionales/publicaciones/presentacion/

Universidad de Colima (2003). Hemeroteca de boletines. Recuperado de http:// www.ucol.mx/boletines/index.php?idn=1673\&mes=12\&dia=2\&year $=2003$

Yurén, T. (2005). Ethos y autoformación en los dispositivos de formación de docentes. En T. Yurén, C. Navia y C. Saenger (coords.). Ethos y autoformación del docente. Análisis de dispositivos de formación de profesores (pp. 19-48). México: Pomares. 